MITSUBISHI ELECTRIC RESEARCH LABORATORIES

http://www.merl.com

\title{
Robust Optimization of EV Charging Schedules in Unregulated Electricity Markets
}

\author{
Korolko, N.; Sahinoglu, Z.
}

TR2015-121 September 2015

\begin{abstract}
In this article, we address the problem of optimal electric vehicle charging in an unregulated electricity market. This problem is known to be highly nonlinear even in the case of fixed electricity prices due to a nonlinear state-of-charge curve representing physical battery limitations. We design tractable formulations for single and multiple EV charging frameworks. In the first part of the paper, we develop a new efficient cutting plane method, that can be used for solving charging optimization problem for both scenarios of known and uncertain electricity prices. The latter scenario with real-time electricity rates is considered in the second part of the paper. We obtain robust optimization counterparts of the nominal charging problems that are particularly important from an economic perspective when budget constraints are strictly enforced. New robust formulations are proven to be tractable. Moreover, computational experiments illustrate that a decision maker can find solutions that are close to optimal in terms of the corresponding objective values, and robust with respect to uncertain electricity prices.
\end{abstract}

2015 IEEE Transactions on Smart Grid

\begin{abstract}
This work may not be copied or reproduced in whole or in part for any commercial purpose. Permission to copy in whole or in part without payment of fee is granted for nonprofit educational and research purposes provided that all such whole or partial copies include the following: a notice that such copying is by permission of Mitsubishi Electric Research Laboratories, Inc.; an acknowledgment of the authors and individual contributions to the work; and all applicable portions of the copyright notice. Copying, reproduction, or republishing for any other purpose shall require a license with payment of fee to Mitsubishi Electric Research Laboratories, Inc. All rights reserved.
\end{abstract}





\title{
Robust Optimization of EV Charging Schedules in Unregulated Electricity Markets
}

\author{
Nikita Korolko, Zafer Sahinoglu, Senior Member, IEEE
}

\begin{abstract}
In this article, we address the problem of optimal electric vehicle charging in an unregulated electricity market. This problem is known to be highly nonlinear even in the case of fixed electricity prices due to a nonlinear state-of-charge curve representing physical battery limitations. We design tractable formulations for single and multiple EV charging frameworks. In the first part of the paper, we develop a new efficient cutting plane method, that can be used for solving charging optimization problem for both scenarios of known and uncertain electricity prices. The latter scenario with real-time electricity rates is considered in the second part of the paper. We obtain robust optimization counterparts of the nominal charging problems that are particularly important from an economic perspective when budget constraints are strictly enforced. New robust formulations are proven to be tractable. Moreover, computational experiments illustrate that a decision maker can find solutions that are close to optimal in terms of the corresponding objective values, and robust with respect to uncertain electricity prices.
\end{abstract}

Index Terms-Electric vehicle, robust optimization, charging schedule, state-of-charge curve.

\section{NOMENCLATURE}

A. Indices

$t$ Time index

$v \quad \mathrm{EV}$ index

$l \quad$ Time shift index

B. Sets
$T \quad$ Feasible set of a nominal problem (1)
$U_{i}(\Gamma) \quad$ Uncertainty set with robustness level $\Gamma$
$C_{l} \quad$ Charging shift

\section{Parameters}

$m_{t} \quad$ Electricity unit price at time $t$

$S_{i n l} \quad$ Initial battery state-of-charge (SoC)

$Q_{r} \quad$ Battery capacity divided by time resolution $\Delta t$

$\Gamma \quad$ Robustness level

$B \quad$ Charging budget

$z^{*} \quad$ Optimal charging cost

$\theta^{*} \quad$ Optimal battery charge level

$\nu(U) \quad$ Empirical probability that price vector is an element of $U$

$\mu(U) \quad$ Empirical probability that charging policy $\mathbf{p}(U)$ does not violate budget constraint

$V \quad$ EV fleet size

$L \quad$ The number of charging shifts

N. Korolko is with the Operations Research Center, Massachusetts Institute of Technology, Cambridge, MA, 02139 USA, e-mail: korolko@mit.edu

Z. Sahinoglu is with Mitsubishi Electric Research Laboratories, Cambridge, MA, 02139 USA, e-mail: zafer@merl.com

\section{Variables}

$\begin{array}{ll}p_{t} & \text { Charging level at time } t \\ \mathbf{p}^{*} & \text { Testing point } \\ \hat{\mathbf{p}} & \text { Projection point } \\ \mathbf{y} & \text { Vector of dual variables } \\ \mathbf{x} & \text { Simulated vector of electricity prices } \\ Y_{l}^{v} & \text { Binary assignment indicator of EV } v \text { to shift } C_{l}\end{array}$

\section{INTRODUCTION}

The rapidly growing market of electric vehicles $(\mathrm{EV})$ is driven by environmentally friendly zero-emission engines with low noise level, lower operating costs and other social and economic benefits [1]. The global goals of improving air quality and emission reduction [2] suggest even higher penetration of EVs in both corporate and residential automobile market segments. Despite the fact that in most parts of the world the electricity cost per mile of an EV is approximately onethird to one-quarter the cost of gasoline [3], the problem of minimizing the EV charging expense is still important for both commercial and individual EV users.

The problem of finding the optimal charging schedule can be very different for various electricity pricing mechanisms. The typical electricity tariff structures include fixed, time-ofuse (TOU), and real-time electricity rates. The first scenario implies a constant energy charge that does not depend on the time of consumption. The TOU price can be represented as a piecewise-constant function of time with off-peak and on-peak prices, while the real-time pricing mechanism assumes that customers pay electricity supply rates that continuously vary by the hour [4]. For a fixed electricity rate scenario, total EV charging cost does not depend on time, therefore all feasible charging schemes have the same price. On the other hand, the associated optimization problems become more complicated for the cases of TOU and real-time pricing mechanisms. Indeed, the TOU instance implies that the vector of prices does not consist of equal entries, while the real-time rates instance suggests that the price vector is not known in advance and should be somehow estimated.

Another inherent property of charging optimization problem is nonlinearity of its constraints [5]. In order to reduce the life loss of EV battery, the charging power should not exceed a certain confined value defined by a nonlinear concave function of battery SoC. Although incorporation of nonlinear SoC curve into optimization problem does not affect convexity of the feasible region, it does impair its tractability.

Several studies have already been done to solve simplified versions of the charging optimization problem. For instance, there are approaches presented in [4] - [8] that are valid under 
assumption of known and fixed electricity prices. More precisely, Qian et. al [4] consider a piecewise-constant approximation of nonlinear SoC curves, which makes the optimization problem linear. A comparison between linear and quadratic approximations of the problem is given in [6]. Reference [7] describes another linearized version of the optimal EV charging problem that incorporates possibility to perform vehicleto-grid delivery. Cao et. al [5] suggest a heuristic method for minimizing the charging cost based on iterative transition of a small energy portion from a high-price period to a low-price period. An optimization model for charging and discharging with given driving patterns of the fleet of EVs is presented in [8]. But the formulation assumes that maximum charging level is constant, and electricity prices are either known or predicted via linear regression as a function of load aggregator demand.

EV charging models based on uncertain electricity pricing are considered in [9] - [14]. Namely, there is a simple formula for EV charging rate with vehicle-to-grid capability presented in [9], but the optimality of the suggested formula is not discussed. Reference [10] introduces an optimization of the charging and discharging of EVs considering only a piecewiselinear dependence between a power system load level and electricity price. The fleet aggregator perspective that manages charging and purchasing in the day-ahead electricity market is considered in [11]. The authors develop a bidding strategy minimizing transaction costs and taking into account other market participants. Reference [12] presents decentralized approach to EV charging with objective of load demand valley filling and assuming collaboration between EVs and a utility company. Discrete cost optimization method similar to [5] based on Markovian chains is introduced in [13]. In this model the price uncertainty depends on only historic maximum price of electricity purchasing, and the connection between EVs in the fleet is stated, but not incorporated to Markov process. Robust optimization (RO) EV charging model with technical power grid constraints is described in [14]. But only one specific type of price uncertainty set is considered, nonlinear SoC battery limitations are not included and tractability of suggested optimization problem is not assessed.

In our study, we derive a generic formulation of optimal EV charge scheduling with nonlinear constraints and uncertainty of the price of electricity where applicable. Section 2 develops a tractable method of solving a nominal problem with fixed electricity prices. The description of the method involves the proof of convexity of the feasible set, projection formulas, as well as separating oracle for this nonlinear set (i.e. a procedure that for any given point either tells that it belongs to a set or returns a hyperplane separating a point from the set). This tool necessary for implementation of a cutting plane algorithm, which serves as an efficient method for solving the introduced charging problem.

Section 3 discusses RO approach to the EV charging problem, when electricity prices do not have to be known in advance, but rather belong to some uncertainty set. This perspective is useful for budget planning or finding a charging schedule that does not exceed some budget constraints. In this section, we prove that the robust counterparts are still tractable, since the cutting plane algorithm can be extended for the case of uncertain electricity prices.

The numerical experiments based on real-world electricity pricing data are presented in Section 4. The main objective of this analysis is to compare optimal charging schedules and their performance for a nominal optimization problem and its robust counterparts with various uncertainty sets. Computational experiments demonstrate that it is possible to find robust charging schedules that simultaneously have two merits. They yield objective values that are close to optimal, and they are stable with respect to uncertain electricity rates.

A load aggregator perspective and corresponding extensions of the EV fleet charging optimization problems are designed in Section 5. Finally, Section 6 discusses universalism and computational efficiency of the suggested cutting plane algorithm.

\section{OPTIMIZATION PROBLEM AND ITS TRACTABILITY}

\section{A. Problem description}

The EV charging optimization problem can be formulated as finding a schedule $P(t)$ that minimizes the total cost

$$
C=\int_{t_{0}}^{t_{0}+T} m(t) P(t) d t,
$$

where $m(t)$ is the unit electricity price at time $t$, and $\left[t_{0} ; t_{0}+T\right]$ is a time interval of charging [5]. In order to prevent battery damage, the charging power $P(t)$ is upper bounded as described by inequality

$$
0 \leq P(t) \leq P_{\max }(t) \quad \forall t \in\left[t_{0} ; t_{0}+T\right] .
$$

The maximum charging level $P_{\max }(t)$ in practice can be expressed as a concave non-increasing function of the current SoC $S(t)$ according to the formula from [5], [15]:

$$
P_{\text {max }}(t)=f(S(t)) \text {. }
$$

In this paper we incorporate the smooth nonlinear SoC curve $f(S)$ and its derivative $f^{\prime}(S)$ presented in Fig. 1. This particular function $f(S)$ serves as an example and corresponds to an advanced AC Level III charging system. However, all results of the study remain valid for any non-increasing, quasiconcave function $f(S)$ that does not even have to be differentiable.

The charging level $P(t)$ can be also limited from above by the maximum power $P_{\text {user }}$ set by EV user and the maximum power $P_{\text {charger }}$ that EV charger can output. Upper bounding of $P(t)$ is also necessary for economic reasons to limit the cost of demand charge imposed by utility firms on EV chargers. Since these restrictions can be expressed in terms of simple linear inequalities (or incorporated to the structure of SoC function $f(S)$ ), we do not explicitly add them to our model.

The total charging demand of an EV user with the initial SoC $S_{i n l}$ and rated capacity $\bar{Q}_{r}$ is given by equality

$$
\int_{t_{0}}^{t_{0}+T} P(t) d t=\left(1-S_{i n l}\right) \bar{Q}_{r} .
$$

The discrete counterpart of the continuous optimization problem with time resolution $\Delta t$ can be expressed as follows:

$$
\min _{\mathbf{p}} \sum_{t=0}^{N} m_{t} p_{t}
$$




$$
\begin{array}{ll}
\text { s.t. } \quad 0 \leq p_{0} \leq f\left(S_{\text {inl }}\right) \\
0 \leq p_{t} \leq f\left(S_{i n l}+\frac{1}{Q_{r}} \sum_{k=0}^{t-1} p_{k}\right), \quad \forall t=1 \ldots N \\
\sum_{t=0}^{N} p_{t}=\left(1-S_{\text {inl }}\right) Q_{r},
\end{array}
$$

where a common positive factor $\Delta t$ in objective (1a) is ommited and $S_{i n l}, f\left(S_{i n l}\right), Q_{r}=\bar{Q}_{r} / \Delta t$ are assumed to be known constants. At the same time, components of the cost vector $\mathbf{m}$ can be either fixed and known a priori for the case of a regulated electricity market, or uncertain for a real-time pricing scenario. In this section, we consider a nominal problem with constant components $m_{t}$ in the objective function (1a). The equality (1d) can be generalized to inequality

$$
\alpha \cdot Q_{r} \leq \sum_{t=0}^{N} p_{t} \leq\left(1-S_{i n l}\right) Q_{r}
$$

with $0 \leq \alpha \leq 1-S_{i n l}$, if decision maker wants to charge a battery up to at least $\left(S_{i n l}+\alpha\right) \cdot 100 \%$ of the total rated capacity $\bar{Q}_{r}$.

The feasible region of the nominal optimization problem denoted as $T$ and described by (1b) - (1d) is nonlinear due to a large number of constraints of the type (1c). On the other hand, for any fixed $t=1 \ldots N$ the function $f\left(S_{i n l}+\frac{1}{Q_{r}} \sum_{k=0}^{t-1} p_{k}\right)$ is concave with respect to variables $p_{0}, \ldots, p_{N}$ as a composition of linear and non-increasing concave function $f$. Moreover, the nonlinear upper bound for variable $p_{t}$ in (1c) depends on only variables with lower indices $p_{0}, \ldots, p_{t-1}$. Therefore, each of these constraints defines a convex set which is a hypograph of a concave function $f\left(S_{i n l}+\frac{1}{Q_{r}} \sum_{k=0}^{t-1} p_{k}\right)$. This is why a feasible set $T$ is convex as an intersection of a finite number of convex sets.

\section{B. Cutting plane algorithm}

In order to prove that the nominal problem is tractable we develop a cutting plane algorithm for our special form of the feasible region $T$. The main idea of the method is to solve the nonlinear optimization problem by solving a sequence of linear programming problems. We start with finding an optimal solution $\mathbf{p}^{*}$ of a relaxed nominal problem that includes only linear constraints from formulation (1). If $\mathbf{p}^{*}$ satisfies all omitted nonlinear constraints (1c), then $\mathbf{p}^{*}$ is an optimum of the initial problem. If not, we project a testing point $\mathbf{p}^{*}$ onto a convex set $T$ and obtain a projection point $\hat{\mathbf{p}}$. After this, it is necessary to find a supporting hyperplane to the set $T$ at the point $\hat{\mathbf{p}}$. This hyperplane separates testing point $\mathbf{p}^{*}$ from the feasible region $T$. We add the linear inequality defining a halfspace that contains set $T$ and then iterate [18].

The remaining part of this subsection describes membership and separation oracles for the set $T$, as well as a projection method that we incorporate into the cutting plane algorithm.

Let us assume that after some iteration of the algorithm there is a testing point $\mathbf{p}^{*}=\left(p_{0}^{*}, p_{1}^{*}, \ldots, p_{N}^{*}\right)$ that satisfies nonnegativity constrainsts $p_{t}^{*} \geq 0$ for all $t=0 \ldots N$ and linear constraints (1b) and (1d). In this case, the feasibility of point $\mathbf{p}^{*}$ can be verified by gradual inspection of nonlinear constraints of type (1c). Indeed, we can run a loop with respect to the time index $t=1 \ldots N$, and for each fixed value of $t$ it is easy to check if the elements of $\mathbf{p}^{*}$ satisfy $t$-th constraint of a family (1c).

If all $N$ constraints of type (1c) are satisfied, then the testing point $\mathbf{p}^{*}$ belongs to the feasible set $T$. Otherwise, membership oracle displays the smallest time index $t_{0}$ for which an upper bound of (1c) is violated:

$$
t_{0}=\underset{t=1 \ldots N}{\operatorname{argmin}}\left\{p_{t}^{*}>f\left(S_{i n l}+\frac{1}{Q_{r}} \sum_{k=0}^{t-1} p_{k}^{*}\right)\right\} .
$$

Having found a violated constraint, the projection $\hat{\mathbf{p}}$ of the point $\mathbf{p}^{*}$ onto the boundary of the convex set $T$ is described as follows

$$
\hat{p}_{t}= \begin{cases}p_{t}^{*}, & \text { if } 0 \leq t<t_{0}, \\ f\left(S_{i n l}+\frac{1}{Q_{r}} \sum_{k=0}^{t_{0}-1} p_{k}^{*}\right), & \text { if } t=t_{0}, \\ 0, & \text { if } t_{0}<t \leq N .\end{cases}
$$

Feasibility of point $\hat{\mathbf{p}}$ is due to the definition of time index $t_{0}$. Since inequalities of family (1c) with indices $t=t_{0}, \ldots, N$ are active at $\hat{\mathbf{p}}$, then projection point $\hat{\mathbf{p}}$ belongs to the boundary of the set $T$.

The supporting hyperplane to the set $T$ at the point $\hat{\mathbf{p}}$ is defined by standard equality $\nabla F(\hat{\mathbf{p}})^{\top}(\mathbf{p}-\hat{\mathbf{p}})=0$, where function $F$ is defined as

$$
F\left(p_{0}, \ldots, p_{N}\right)=p_{t_{0}}-f\left(S_{i n l}+\frac{1}{Q_{r}} \sum_{k=0}^{t_{0}-1} p_{k}\right),
$$

and components of its gradient are expressed as below

$$
\frac{\partial F}{\partial p_{t}}(\hat{\mathbf{p}})= \begin{cases}-f^{\prime}(\hat{p}) \frac{1}{Q_{r}} & \text { if } 0 \leq t<t_{0} \\ 1 & \text { if } t=t_{0} \\ 0 & \text { if } t_{0}<t \leq N .\end{cases}
$$

The separating inequality $\nabla F(\hat{\mathbf{p}})^{\top}(\mathbf{p}-\hat{\mathbf{p}}) \leq 0$ does not cut any feasible point of the convex region $T$, while testing point $\mathbf{p}^{*}$ violates it. Indeed,

$$
\begin{aligned}
\nabla F(\hat{\mathbf{p}})^{\top}\left(p^{*}-\hat{p}\right) & =-f^{\prime}(\hat{\mathbf{p}}) \frac{1}{Q_{r}} \sum_{t=0}^{t_{0}-1}\left(p_{t}^{*}-p_{t}^{*}\right) \\
& +1 \cdot\left(p_{t_{0}}^{*}-\hat{p}_{t_{0}}\right)+\sum_{t=t_{0}+1}^{N} 0 \cdot\left(p_{t}^{*}-0\right)>0
\end{aligned}
$$

since the only nonzero term $\left(p_{t_{0}}^{*}-\hat{p}_{t_{0}}\right)$ is strictly positive by the definition of time index $t_{0}$. We add linear separating inequality into the formulation preserving linearity of the optimization problem, and iterate this step. The cutting plane method is an exact algorithm that is guaranteed to find an optimal solution of the nonlinear charging optimization problem [18]. The stopping condition and computational efficiency of the method are discussed in Section 6. 

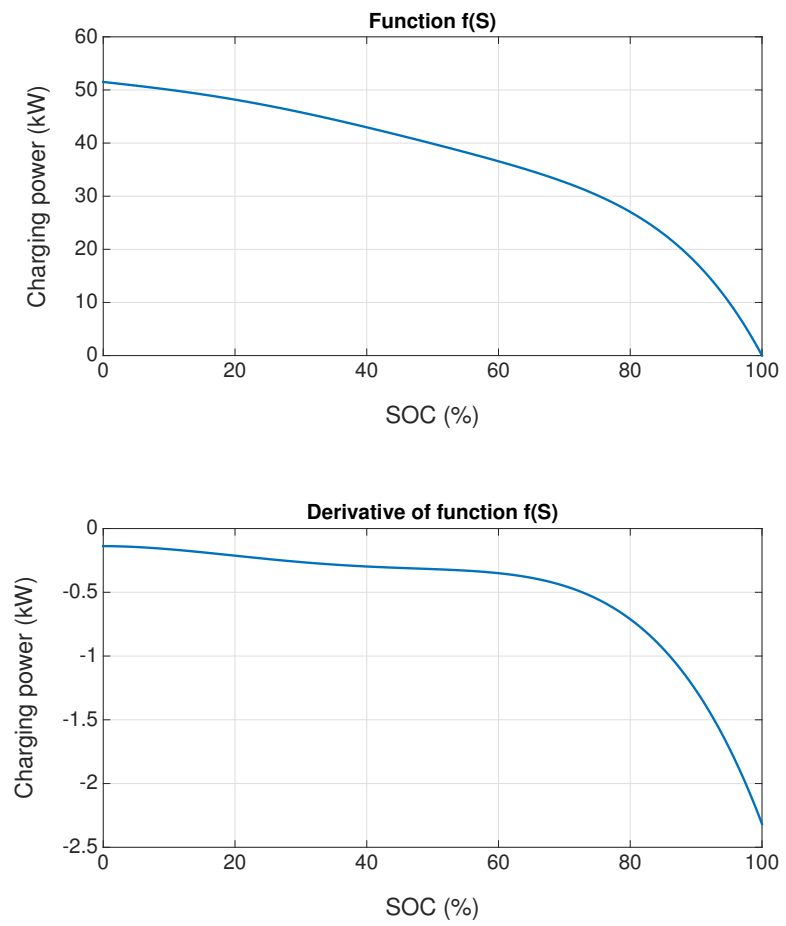

Figure 1. The SoC curve $f(S)$ and its derivative $f^{\prime}(S)$

\section{ROBUST OPTIMIZATION APPROACH}

Wholesale prices fluctuate a lot from one day to another and the variation between low-demand night hours and highdemand business hours can be huge. In this section, we are going to generalize the nominal problem (1) to the case of uncertain electricity prices. This scenario assumes that the cost vector $\mathbf{m}$ in the objective function (1a) is not known in advance, but rather belongs to some uncertainty set $U$ [16], [17]. Hence, components $m_{t}$ for $t=0 \ldots N$ should be treated as uncertain parameters in place of fixed values to represent an unregulated electricity market with real-time pricing mechanisms.

Some basic examples of the uncertainty sets $U$ are listed below:

1) $U_{1}=\left\{\mathbf{m} \in \mathbb{R}^{N+1}: \underline{m}_{t} \leq m_{t} \leq \bar{m}_{t}, t=0 \ldots N\right\}$, where $\underline{m}_{t}$ and $\bar{m}_{t}$ are estimated from historic data lower and upper bounds on the electricity price $m_{t}$ at time $t$.

2) $U_{2}(\Gamma)=\left\{\mathbf{m} \in \mathbb{R}^{N+1}:\left|\frac{m_{t}-\mathbb{E} m_{t}}{\sigma\left(m_{t}\right)}\right| \leq \Gamma, t=0 \ldots N\right\}$ or

3) $U_{3}(\Gamma)=\left\{\mathbf{m} \in \mathbb{R}^{N+1}: \sum_{t=0}^{N}\left|\frac{m_{t}-\mathbb{E} m_{t}}{\sigma\left(m_{t}\right)}\right| \leq \Gamma \sqrt{N+1}\right\}$, where $\mathbb{E} m_{t}$ and $\sigma\left(m_{t}\right)$ are the expected value and standard deviation of a random electricity rate $m_{t}$, respectively. These values could be estimated by statistical methods given some training data. At the same time, a nonnegative parameter $\Gamma$ reflects the level of robustness. Indeed, the bigger the value of $\Gamma$, the larger the uncertainty set $U$. Consequently, the price vector $\mathbf{m}$ is more likely to belong to $U$.

For the RO approach that we develop it is sufficient to assume that the uncertainty set $U$ is a nonempty bounded polyhedron with respect to variables $m_{0}, \ldots, m_{N}$ [17]. Therefore, the following representation holds

$$
U=\left\{\mathbf{m} \in \mathbb{R}^{N+1}: \mathbf{D m} \leq \mathbf{d}\right\}
$$

for some matrix $\mathbf{D} \in \mathbb{R}^{n \times(N+1)}$ and vector $\mathbf{d} \in \mathbb{R}^{n}$. It is easy to see that the uncertainty sets $U_{i}$ for $i=1,2,3$ introduced above can be represented in the form (2).

Given $U$, the robust counterpart of the nominal problem (1) is expressed by the following min-max formulation

$$
z_{\text {rob }}(U)=\min _{\mathbf{p} \in T} \max _{\mathbf{m} \in U} \sum_{t=0}^{N} m_{t} p_{t} .
$$

The decision maker tries to find an optimal charging schedule $\mathbf{p} \in T$ under uncertain electricity prices $m_{0}, \ldots, m_{N}$, that she believes are randomly chosen by nature from the uncertainty set $U$. Moreover, the selection of $\mathbf{p}$ is done against the worst possible scenario, captured by maximization with respect to parameter $\mathbf{m}$.

The robust framework is motivated by economical perspective of the EV charging problem. For instance, if there is a fleet of EVs and an agent is developing a budget plan for charging the fleet, the question of the highest possible charging price is essential when the electricity rates are uncertain. In this case, the robust budget estimation problem (3) yields not only an upper bound on the total charging price $z_{r o b}(U)$, but also the corresponding optimal charging policy $\mathbf{p}_{\text {rob }}^{*}(U)$.

Another example of a practical problem that can be modeled with RO techniques has in some sense a dual nature to the problem (3). Let us assume that the maximum acceptable budget $B$ for charging is given. If the decision maker tries to maximize the total battery charge without violating the budget constraint, we obtain a robust formulation (4).

$$
\begin{array}{cl}
\theta_{\text {rob }}(U)=\max _{\mathbf{p}} & \sum_{t=0}^{N} p_{t} \\
\text { s.t. } & \sum_{t=0}^{N} m_{t} p_{t} \leq B, \quad \forall \mathbf{m} \in U \\
& 0 \leq p_{0} \leq f\left(S_{\text {inl }}\right) \\
& 0 \leq p_{t} \leq f\left(S_{\text {inl }}+\frac{1}{Q_{r}} \sum_{k=0}^{t-1} p_{k}\right), \\
& \forall t=1 \ldots N .
\end{array}
$$

The tractability proofs of semi-infinite programming problems (3) and (4) are developed in the propositions below.

Proposition 1. RO problem (3) with an uncertainty set of the type (2) is equivalent to the computationally tractable nonlinear programming problem (5).

$$
\begin{array}{cl}
\min _{\mathbf{p}, \mathbf{y}, z} & z \\
\text { s.t. } & \mathbf{y}^{\top} \mathbf{d} \leq z \\
& \mathbf{y}^{\top} \mathbf{D}=\mathbf{p}^{\top} \\
& \mathbf{y} \geq \mathbf{0} \\
& \mathbf{p} \in T .
\end{array}
$$

Proof. First of all, we introduce a new auxiliary scalar variable $z$ and represent min-max problem (3) in the following 
form

$$
\begin{array}{ll}
\min _{\mathbf{p}, z} & z \\
\text { s.t. } & \max _{\mathbf{m} \in U} \mathbf{m}^{\top} \mathbf{p} \leq z \\
& \mathbf{p} \in T .
\end{array}
$$

For any fixed value of vector $\mathbf{p}$ we consider the inner linear optimization problem

$$
\begin{array}{cl}
\max _{\mathbf{m}} & \mathbf{m}^{\top} \mathbf{p} \\
\text { s.t. } & \mathbf{D m} \leq \mathbf{d} .
\end{array}
$$

Since the uncertainty set $U$ described by the nonempty polyhedron $\{\mathbf{D m} \leq \mathbf{d}\}$ is assumed to be bounded, then the primal problem $(\mathcal{P})$ is feasible and has a finite optimum. Therefore, by the strong duality theorem [18] the optimal cost of the problem $(\mathcal{P})$ is equal to the optimal cost of the dual problem $(\mathcal{D})$ :

$$
\begin{array}{cl}
\min _{\mathbf{y}} & \mathbf{y}^{\top} \mathbf{d} \\
\text { s.t. } & \mathbf{y}^{\top} \mathbf{D}=\mathbf{p}^{\top} \\
& \mathbf{y} \geq \mathbf{0} .
\end{array}
$$

Hence, the optimization problem (6) can be updated as follows

$$
\begin{array}{cl}
\min _{\mathbf{p}, \mathbf{y} z} & z \\
\text { s.t. } & \min _{\mathbf{y}} \mathbf{y}^{\top} \mathbf{d} \leq z \\
& \mathbf{y}^{\top} \mathbf{D}=\mathbf{p}^{\top} \\
& \mathbf{y} \geq \mathbf{0} \\
& \mathbf{p} \in T .
\end{array}
$$

Finally, it is easy to see that the inner minimization with respect to $\mathbf{y}$ in the problem $\left(6^{\prime}\right)$ can be omitted, what immediately yields the formulation (5).

It is worth mentioning that the latter problem has the same nonlinear constraints as the nominal problem (1). That is why the cutting plane algorithm designed in Section 2 is still applicable, what makes problem (5) tractable.

For the semi-infinite optimization charging problem with a budget constraint (4), the similar duality argument can be developed in order to prove the following proposition.

Proposition 2. RO problem (4) with an uncertainty set of the type (2) is equivalent to the computationally tractable nonlinear programming problem (7).

$$
\begin{array}{cl}
\max _{\mathbf{p}, \mathbf{y}, z} & \sum_{t=0}^{N} p_{t} \\
\text { s.t. } & \mathbf{y}^{\top} \mathbf{d} \leq B \\
& \mathbf{y}^{\top} \mathbf{D}=\mathbf{p}^{\top} \\
& \mathbf{y} \geq \mathbf{0} \\
& 0 \leq p_{0} \leq f\left(S_{i n l}\right) \\
& 0 \leq p_{t} \leq f\left(S_{i n l}+\frac{1}{Q_{r}} \sum_{k=0}^{t-1} p_{k}\right), \forall t=1 \ldots N .
\end{array}
$$

Thus, the highly nonlinear EV charging optimization problem (1) is tractable not only with a fixed cost vector $\mathbf{m}$, but also when electricity prices $m_{0}, \ldots, m_{N}$ are uncertain. The latter scenario can be modeled with a combination of the cutting plane algorithms and robust programming techniques.

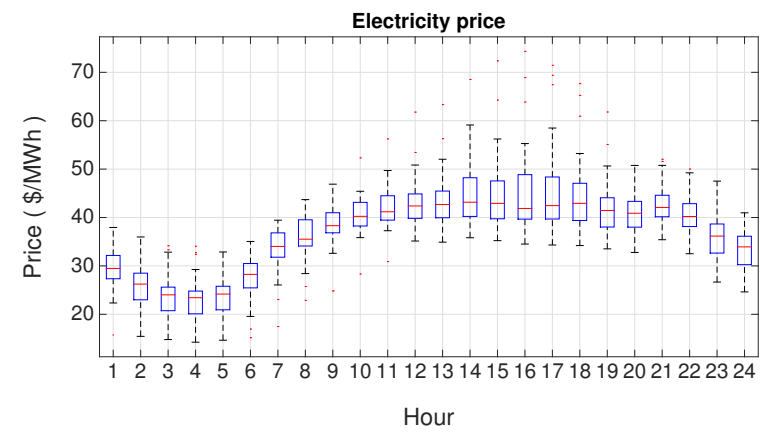

Figure 2. Hourly unit electricity prices

\section{COMPUTATIONAL RESUlTS}

In this section, we compare the performance of two different approaches to the EV charging problem using the real-world electricity pricing data. The first approach with fixed electricity rates is the nominal problem (1) introduced in Section 2. The second $\mathrm{RO}$ framework includes two possible formulations (3) and (4) with various uncertainty sets $U_{i}$ defined in the beginning of Section 3. Two approaches will be compared in terms of some performance indicators, such that the optimal charging price $z_{\text {rob }}^{*}$, the guaranteed total battery charge $\theta_{\text {rob }}^{*}$, and robustness $\mu$ of the charging policy $\mathbf{p}^{*}$ that we will explicitly define below.

From the dataset [19] published by the US National Grid for New York City we derive hourly electricity prices for weekdays of 2 consecutive months: May and June 2014 (Fig. 2). The selected data include the total of $K=43$ observations, where each observation is a vector of the length 24 .

Having assumed that the electricity rate is fixed during any given hour, now it is possible to estimate parameters

$\mathbb{E} m_{j}=\frac{\sum_{k=1}^{K} m_{j}(k)}{K}$ and $\sigma^{2}\left(m_{j}\right)=\frac{\sum_{k=1}^{K}\left(m_{j}(k)-\mathbb{E} m_{j}\right)^{2}}{K-1}$,

for all $j=1 \ldots 24$, where $m_{j}(k)$ denotes an electricity price of the $j$-th hour in the $k$-th observation.

Additionally, for each of the hours $j=1, \ldots, 24$ we test a hypothesis that random electricity rate $m_{j}$ is a normal random variable with parameters $\mathbb{E} m_{j}$ and $\sigma^{2}\left(m_{j}\right)$. More precisely, Kolmogorov-Smirnov statistical method [20] allows us to find a minimum confidence level $f_{j} \in[0,1]$ with which a conjecture $H_{0}=\left\{m_{j} \in \mathbb{N}\left(\mathbb{E} m_{j}, \sigma^{2}\left(m_{j}\right)\right)\right\}$ can be accepted. We use MATLAB function kstest [21] and derive that the vector $\mathbf{f}=\left(f_{1}, \ldots, f_{24}\right)$ consists of $f_{j}=0.99$ for all $j=1 \ldots 24$.

The computation of vector $\mathbf{f}$ is necessary for quality estimation of the uncertainty sets $U$. Indeed, given that components of the vector $f$ have high values, now it is possible to sample random vector

$$
\begin{aligned}
\mathbf{x}=\left(x_{1}, \ldots, x_{24}\right), \\
\quad \text { s.t. } x_{j} \in \mathbb{N}\left(\mathbb{E} m_{j}, \sigma^{2}\left(m_{j}\right)\right), \text { for } j=1, \ldots, 24,
\end{aligned}
$$

and check if $\mathbf{x}$ is an element of a given uncertainty set $U$. The higher the empirical probability $\nu(U)$ that $\mathbf{x} \in U$, the better the reliability of the uncertainty set $U$. 


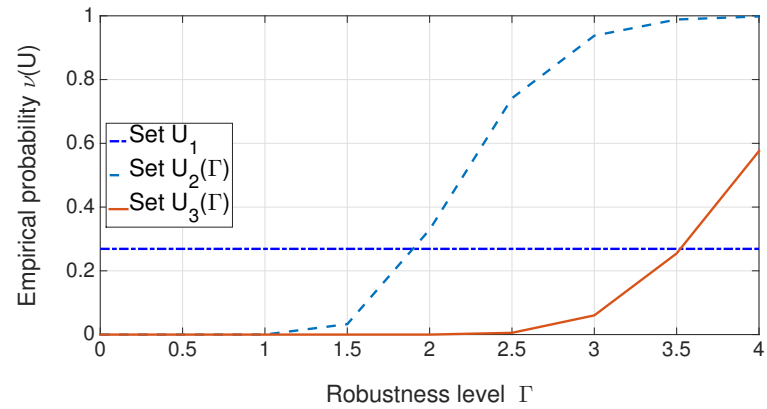

Figure 3. Empirical probability $\nu\left(U_{i}\right), i=1,2,3$ that simulated price is an element of uncertainty set $U_{i}(\Gamma)$

The time interval of possible charging $\left[t_{0} ; t_{0}+T\right]$ in our experiments is assumed to be equal to $[0 h, 24 h]$. We discretize this interval at a sampling frequency of 1 observation per minute, what implies that the total number of time periods $N$ in the model is equal to $1 \cdot 60 \cdot 24=1,400$. For both of the computational experiments considered below, the rate capacity is assumed to be equal to $Q_{r}=29.07 \mathrm{kWh}$ [4] and the initial SoC $S_{i n l}=0.2$.

\section{Experiment A. Robust budget estimation}

In this experiment, we consider RO problem of type (3) for various uncertainty sets. We solve this min-max problem using a duality method suggested in proposition 1 . For different sets $U_{i}, i=1,2,3$ and levels of robustness $\Gamma \in[0,4]$ the following descriptive characteristics are computed:

- The empirical probability $\nu(U)$. This value is an estimation of the probability that the uncertain vector $\mathbf{m}$ is indeed an element of the set $U$. We calculate $\nu(U)$ based on 10,000 simulations with respect to random vector $\mathbf{x}$ with normal components defined by (8). Higher values of the indicator $\nu(U)$ correspond to more robust selections of uncertainty sets $U$. Reliability of sets $U_{i}, i=1,2,3$ as a function of robustness parameter $\Gamma$ is presented in Fig. 3.

- The optimal cost $z_{\text {rob }}^{*}(U)$ (3), that is equal to the robust budget estimate. In other words, $z_{\text {rob }}^{*}(U)$ is an upper bound on the charging cost given that the unknown electricity price vector $\mathbf{m}$ is randomly selected from the suggested uncertainty set $U$. The robust charging costs $z_{\text {rob }}^{*}(U)$ are plotted in Fig. 4.

It is worth mentioning that the nominal problem (1) with vector $\mathbf{m}$ substituted with its expected value $\mathbb{E} \mathbf{m}$ is a special case of robust formulation (3) with uncertainty set $U_{2}$ and $\Gamma=0$. The optimal cost of the nominal problem

$$
z_{\text {nom }}^{*}=z_{\text {rob }}^{*}\left(U_{2}(0)\right)
$$

serves as a benchmark for other robust charging scenarios with the corresponding costs $z_{\text {rob }}^{*}(U)$.

The optimal nominal value $z_{\text {nom }}^{*}$ obtained from Fig. 4 means that the average EV charging cost is equal to $\$ 0.535$. On the other hand, with probability 1 this cost is exceeded according to the simulation results (Fig. 3) and since uncertainty set $U_{2}(0)$ consists of only single point. Therefore, a

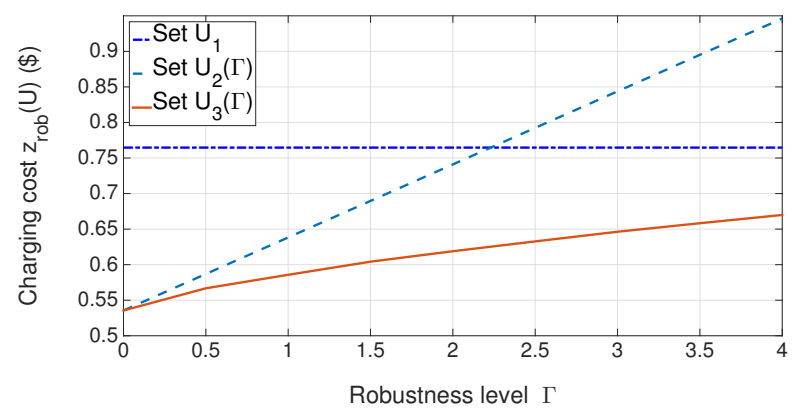

Figure 4. Robust charging cost estimation $z_{\text {rob }}^{*}\left(U_{i}\right), i=1,2,3$ as a function of $\Gamma$

benchmark $z_{\text {nom }}^{*}=0.535$ is not acceptable for robust budget planning.

From Fig. 3 we observe that the reliability of uncertainty sets grows when $\Gamma$ increases. Indeed, it is easy to see that the sets $U_{i}(\Gamma), i=2,3$ become larger as $\Gamma$ goes from 0 to 4 . For instance, a probability that uncertain electricity price vector $\mathbf{m}$ is an element of $U_{2}(3)$ is reasonably high and equals $95 \%$.

At the same time, Fig. 4 demonstrates that the robust budget estimate is also an increasing function of parameter $\Gamma$, since for larger values of argument $\Gamma$ we want to be protected against the worst case of a wider set of scenarios. The implementation of the same uncertainty set $U_{2}(3)$ yields an upper bound on the charging cost that is equal to $\$ 0.843$ that holds with probability $\nu\left(U_{2}(3)\right)=95 \%$ calculated above.

\section{Experiment B. Robust charging with a budget constraint}

The second computational experiment illustrates the robust charging problem with the imposed budget constraint (4). We fix the value of maximum acceptable charging budget $B=\$ 0.7$. The tractability of this semi-infinite nonlinear optimization problem is due to proposition 2. Analogously to the first experiment, we consider different uncertainty sets $U$ and robustness levels $\Gamma$. The performance indicators are:

- The optimal cost value $\theta_{\text {rob }}^{*}(U)$ of problem (4) that is equal to the total guaranteed amount of electricity that we can purchase during the charging without violating the budget constraint. This lower bound $\theta_{r o b}^{*}(U)$ is calculated under assumption that uncertain electricity rates are randomly selected from the set $U$. The exact values of the guaranteed battery charges $\theta_{\text {rob }}^{*}(U)$ are presented in Fig. 5.

- The empirical probability $\mu(U)$ that the optimal solution $\mathbf{p}^{*}(U)$ of the problem (4) is feasible. More precisely, after the optimal charging schedule $\mathbf{p}^{*}(U)$ is found, we simulate vector $\mathbf{x} 10,000$ times according to its definition (8). For each randomly generated $\mathbf{x}$, it is necessary to check if the budget constraint

$$
\sum_{t=0}^{N} m_{t} p_{t}^{*}(U) \leq B
$$

holds, where the charging policy $\mathbf{p}^{*}(U)$ is fixed, and the electricity price vector $\mathbf{m}$ is substituted with vector $\mathbf{x}$. 


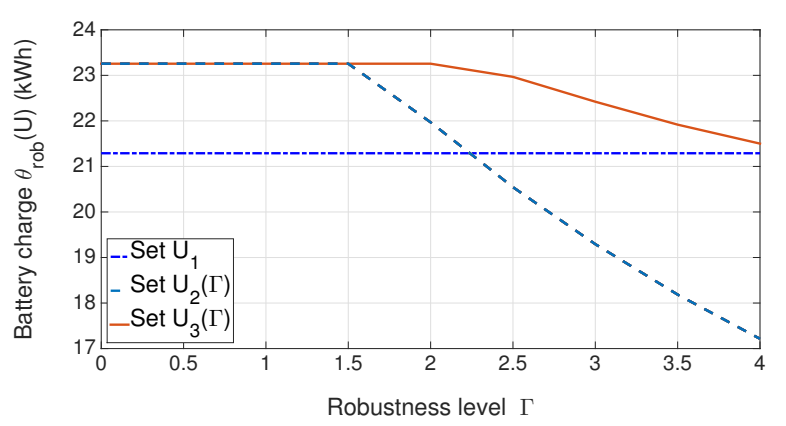

Figure 5. Guaranteed battery charge $\theta_{\text {rob }}^{*}\left(U_{i}\right), i=1,2,3$ as a function of $\Gamma$

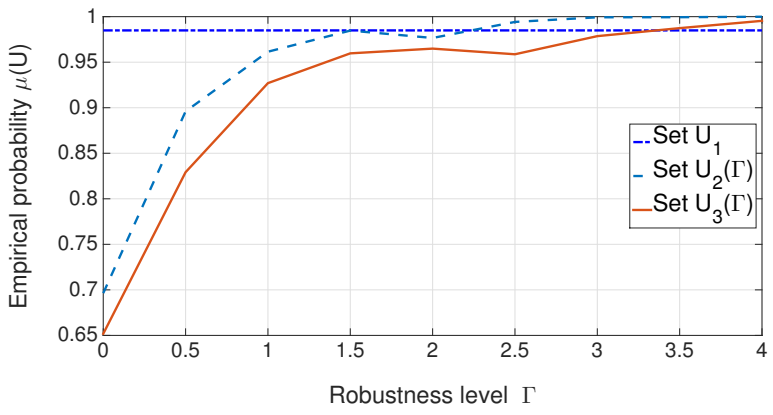

Figure 6. Empirical probability $\mu\left(U_{i}\right), i=1,2,3$ of solution feasibility as a function of $\Gamma$

The portion of scenarios when this inequality holds is equal to $\mu(U)$. The dependence between reliability $\mu(U)$ of suggested charging schedule $\mathbf{p}^{*}(U)$ and parameter $\Gamma$ is illustrated in Fig. 6.

First of all, it is easy to see that a maximum possible value of $\theta_{\text {rob }}^{*}(U)$ presented in Fig. 5 is equal to

$$
\left(1-S_{\text {inl }}\right) \bar{Q}_{r}=(1-0.2) \cdot 29.07=23.35 \mathrm{kWh}
$$

despite the fact that the constraint

$$
\sum_{t=0}^{N} p_{t}=\left(1-S_{i n l}\right) Q_{r}
$$

is removed from the formulation (4). Indeed, when the total charge $\sum_{k=0}^{t-1} p_{k}$ reaches the level $\left(1-S_{i n l}\right) Q_{r}$ for some $t$, the battery becomes fully charged and all the remaining variables $p_{k}, k \geq t$ will be forced to zero since $f(1)=0$.

From Fig. 5 it is easy to see that implementation of uncertainty sets $U_{i}(\Gamma)$ for $i=2,3$ and small values of $\Gamma \leq 1.5$ yields charging schedules $\mathbf{p}^{*}(U)$ that fully recharge the battery. On the other hand, the probability $\mu(U)$ that the budget constraint will not be violated can be as low as $65.2 \%$ what can be observed from Fig. 6 .

At the same time, for instance, if the uncertainty set $U_{2}(2)$ is incorporated to the formulation (4), then we obtain the charging schedule $\mathbf{p}^{*}$ with the total charge $\theta_{\text {rob }}^{*}=21.97 \mathrm{kWh}$ and probability $97.8 \%$ that we do not exceed budget $B=\$ 7.0$. In this scenario, the cost function $\theta_{\text {rob }}^{*}$ is only $\frac{23.35-21.97}{23.35}=5.9 \%$ suboptimal, while the probability $\mu\left(U_{2}(2)\right)$ that solution $\mathbf{p}^{*}$ is feasible is reasonably high and close to $100 \%$.
The latter example demonstrates only one possible choice of decision $\mathbf{p}^{*}(U)$ that can be made taking into consideration a trade-off between optimality $\theta_{\text {rob }}^{*}(U)$ and robustness $\mu(U)$. Other possible selections are presented in Fig. 5 and 6.

\section{Generalizations of FORMUlations}

In order to address a larger set of possible real-world scenarios with additional practical constraints, in this section we consider generalizations of optimization problems (1), (3) and (4). First of all, EV charging problem under uncertain electricity prices is more relevant for a fleet of vehicles associated with a common load aggregator rather than a single $\mathrm{EV}$. The reason is that load aggregator has access to wholesale market with fluctuating prices, which is rarely the case for individual EVs. This extension can be modeled by introduction of the second car index $v=1 \ldots V$ for decision variables $p_{t}$ from (1) - (7), where $V$ is the size of the fleet.

The second practical limitation is a length of the charging period during which EV is attached to a charger. Since this period is rarely equal to 24 hours, we introduce the concept of fleet charging shifts $C_{l}, l=1 \ldots L$ that can be described as arbitrary subsets of the global optimization time horizon $\{1, \ldots, N\}$. Moreover, in order to be able to assign EVs $v=1 \ldots V$ to charging shifts $C_{l}, l=1 \ldots L$, we introduce additional binary decision variables

$$
Y_{l}^{v}= \begin{cases}1, & \text { if } v \text { can be charged only during time slot } C_{l}, \\ 0, & \text { otherwise. }\end{cases}
$$

Having defined necessary sets and variables, now it is possible to consider an extended version of charging optimization problem (1) for a decision maker managing the fleet of EVs.

$$
\begin{array}{ll}
\min _{\mathbf{p}, \mathbf{Y}} & \sum_{v=1}^{V} \sum_{t=0}^{N} m_{t} p_{t}^{v} \\
\text { s.t. } & 0 \leq p_{0}^{v} \leq f^{v}\left(S_{i n l}^{v}\right), \quad \forall v=1 \ldots V \\
& 0 \leq p_{t}^{v} \leq f^{v}\left(S_{i n l}^{v}+\frac{1}{Q_{r}^{v}} \sum_{k=0}^{t-1} p_{k}^{v}\right), \quad \forall t \leq N, \forall v \\
& \sum_{t=0}^{N} p_{t}^{v}=\left(1-S_{i n l}^{v}\right) Q_{r}^{v}, \quad \forall v=1 \ldots V \\
& \alpha_{l}^{1} \leq \sum_{v=1}^{V} Y_{l}^{v} \leq \alpha_{l}^{2}, \quad \forall l=1 \ldots L \\
& \sum_{t \notin C_{l}} p_{t}^{v} \leq M \cdot\left(1-Y_{l}^{v}\right), \quad \forall v=1 \ldots V \\
& \sum_{l=1}^{L} Y_{l}^{v}=1, \quad \forall l=1 \ldots L \\
& Y_{l}^{v} \in\{0,1\}, \quad \forall l=1 \ldots L, \quad \forall v=1 \ldots V .
\end{array}
$$

Constraints (9b) - (9d) represent physical charging limitations for each of the EVs $v=1 \ldots V$. Parameters $S_{i n t l}^{v}, Q_{r}^{v}$ and SoC functions $f^{v}(S)$ have the same meaning as before, but now they can vary across the fleet. Possible upper and lower bounds ( $\alpha_{l}^{2}$ and $\alpha_{l}^{1}$, respectively) on the number of EVs that can be charged during a time shift $C_{l}$ are described by 
inequalities (9e). The upper bounds $\alpha_{l}^{2}$ may be also adjusted to represent the total demand charge limitation for the entire fleet. Conditions (9f) prohibit charging of the EVs outside time shifts to which they are assigned, given that constant $M$ is large enough. It is sufficient to take $M$ to be equal to $N \cdot \max _{v=1 \ldots V} f^{v}(0)$. Finally, each $\mathrm{EV}$ is assigned to exactly one time shift $C_{l}$ due to $(9 \mathrm{~g})$. Mixed integer nonlinear optimization formulation (MINLP) (9) models a centralized approach to EV fleet charging problem with several time shifts.

The extension of the dual problem (4) with the objective to maximize the total charging level of the EV fleet without exceeding a given global budget $B$ is almost identical to formulation (9):

$$
\begin{array}{ll}
\max _{\mathbf{p}, \mathbf{Y}} & \sum_{v=1}^{V} \sum_{t=0}^{N} p_{t}^{v} \\
\text { s.t. } & \sum_{v=1}^{V} \sum_{t=0}^{N} m_{t} p_{t}^{v} \leq B
\end{array}
$$

(9b), (9c), (9e) - (9h).

It is worth mentioning, that both nominal MINLPs (9) and (10) with fixed and known electricity prices $\mathbf{m}$ can be generalized to the case of uncertain prices via RO approach presented in Section 3. Indeed, duality technique from propositions 1 and 2 is still valid in spite of introduction of binary variables $Y_{l}^{v}$. This is due to the fact that uncertain parameters $m_{t}, t=$ $0 \ldots N$ are multiplied only by continuous variables $p_{t}^{v}$.

\section{Cutting Plane Method EFFiCienCy}

In this section, we discuss reasoning behind the suggested cutting plane algorithm as well as its comuptational performance.

The first benefit of implementing the algorithm from Section 2 is its universalism. It is applicable to all types of EV charging optimization problems described above. These problems include nominal and robust formulations for both single EV scenario (NLP) and a framework of the EV fleet (MINLP). Indeed, despite the nonconvexity of a feasible set in the latter case, nonlinear optimization problems (9) and (10) with discrete variables $Y_{l}^{v}$ are still solvable by the suggested cutting plane method. The main idea is that feasibility verification of any testing point $\mathbf{p}^{*}=\left(\left(\mathbf{p}^{1}\right)^{*}, \ldots,\left(\mathbf{p}^{V}\right)^{*}\right)$ with respect to nonlinear constraints (9b) and (9c) can be performed independently for each sub-vector $\left(\mathbf{p}^{v}\right)^{*}, v=1 \ldots V$ exatcly as described in subsection 2B.

Moreover, the proposed algorithm does not require SoC functions $f^{v}, v=1 \ldots V$ to be differentiable [22]. It can also handle optimization problems with quasiconvex feasible sets, which are possible for less regular battery chemistry than in the case presented in Fig.1.

The second major advantage of the cutting plane method is computational efficiency. The design of the proposed algorithm exploits specific structure of nonlinear constraints $(9 b),(9 c)$ and therefore significantly outperforms generic off-the-shelf nonlinear solvers. The following set of experiments explicilty illustrates practical benefits of the suggested technique.

In the first two lines of Table 1 we report computational times, number of cuts and iterations of three solvers for the
Table I

COMPUTATIONAL PERFORMANCE OF CUTTING PLANE METHOD

\begin{tabular}{|l|c|c|c|c|c|c|c|}
\hline \multirow{2}{*}{ Exp. } & \multirow{2}{*}{ Type } & \multicolumn{2}{|c|}{ Cut. plane alg. } & \multicolumn{2}{c|}{ Ipopt } & \multicolumn{2}{c|}{ Bonmin } \\
\cline { 3 - 8 } & & Time & Cuts & Time & Iter & Time & Iter \\
\hline \hline 1. (A) & NLP & $9 \mathrm{~s}$ & 32 & $1560 \mathrm{~s}$ & 84 & $283 \mathrm{~s}$ & 18 \\
\hline 2. (B) & NLP & $13 \mathrm{~s}$ & 28 & $975 \mathrm{~s}$ & 89 & $69 \mathrm{~s}$ & 13 \\
\hline 3. (9) & MINLP & $0.37 \mathrm{~h}$ & 4939 & NA & NA & $41.1 \mathrm{~h}$ & $2 \cdot 10^{5}$ \\
\hline 4. (10) & MINLP & $2.23 \mathrm{~h}$ & 7160 & NA & NA & $17.5 \mathrm{~h}$ & $9 \cdot 10^{4}$ \\
\hline
\end{tabular}

experiments A and B described in Section 4 with uncertainty set $U_{1}$. Even the case of a single $\mathrm{EV}$ and a reasonable number of decision variables $(1,440)$ in RO problems (5) and (7) demonstrates the remarkable advantage of the cutting plane approach.

This edge becomes even larger for the case of multiple EVs. Experiment 3 represents solution characteristics of robust counterpart of MINLP (9) with the following values of parameters: fleet size $V=40 ; L=3$ disjoint 8-hours time shifts $C_{l}$; constants $S_{i n l}^{v}=0.2, \bar{Q}_{r}^{v}=29.07$ and SoC functions $f^{v}(S)=f(S)$ are equal for all EVs $v$ in a campaign; uncertainty set is $U_{1}$; upper and lower bounds in inequalities (9e) are $0.5 \mathrm{~V}$ and $0.25 \mathrm{~V}$, respectively. Experiment 4 corresponds to a robust counterpart of (10) with a global budget constant $B=\$ 0.7 \mathrm{~V}$ and the rest of parameters having identical values as in experiment 3 .

The cutting plane algorithm was implemented in Julia/JuMP environment [23] solved by Gurobi Optimizer 6.0.4 and running on Intel Xeon E5440 $(2.83 \mathrm{GHz})$ with 8 cores and 32GB RAM. The perfomance of the method was compared with COIN-OR interior-point and branch-and-bound nonlinear solvers Ipopt 3.12 [24] and Bonmin 1.8 [25]. The tolerance of $0.5 \%$ was chosen to be a stopping condition for all experiments. In all considered instances the suggested iteration method was able to find the optimal solution much faster than off-the-shelf nonlinear solvers.

\section{CONCLUSION}

This work addresses optimum scheduling of single and multiple EV charging for both fixed and uncertain electricity prices. The first feature of this type of problem is nonlinearity. The upper bound on a charging level is represented by a nonlinear concave function of SoC. Despite the incorporation of discrete variables and nonlinear constraints to the model, both membership and linear separating oracles are developed for this set. The latter tools allow us to design an efficient cutting plane algorithm for practical solving of the highly nonlinear EV charging problems.

A major advantage of the suggested cutting plane algorithm is that it can be applied even for the case when electricity rates are not fixed values, but rather uncertain parameters of the optimization problem. This scenario represents an unregulated electricity market with more complex pricing mechanisms than markets with constant or TOU prices that are known in advance. One possible way to handle the electricity price uncertainty is to consider robust counterparts of the nominal 
problems. This approach is important for robust budget estimation of load aggregator and finding a charging schedule that does not violate a given budget constraint. In the second part of the paper, we obtain robust counterparts and prove their tractability.

The numerical experiments demonstrate the advantages of RO framework. We consider two possible optimization problems and corresponding trade-offs between optimality of the cost function and robustness of charging policies. In both cases, we have found feasible solutions that are not far from being optimal in terms of the objective value, but at the same time these robust charging schedules are stable with respect to uncertain electricity prices.

\section{REFERENCES}

[1] Alternative Fuel Data Center, "Benefits and considerations of electricity as a vehicle fuel" [Online]. Available: http://www.afdc.energy.gov/fuels/ electricity_benefits.html [Sep 1, 2015]

[2] United Nations Quantified Economy-Wide Emissions Targets for 2020 [Online]. Available: http://unfccc.int/meetings/copenhagen_dec_ 2009/items/5264.php [Sep 1, 2015]

[3] Idaho National Laboratory, "Advanced Vehicle Testing Activity" [Online]. Available: http://avt.inel.gov/pdf/fsev/costs.pdf [Sep 1, 2015]

[4] K. Qian et al., "Modeling of load demand due to EV battery charging in distribution systems," in IEEE Trans. Power Systems, vol.26, no.2, pp. 802-810, May 2011.

[5] Y. Cao et al., "An optimized EV charging model considering TOU price and SOC curve," in IEEE Trans. Smart Grid, vol.3, no.1, pp. 388-393, March 2012.

[6] O. Sundstrom, C. Binding, "Optimization Methods to Plan the Charging of Electric Vehicle Fleets," in Int. Conf. on Control, Commun. and Power Eng., pp. 28-29, 2010

[7] S. You et. al, "Numerical comparison of optimal charging schemes for Electric Vehicles," in IEEE Power Energy Soc. Gen. Meeting, pp. 1-6, 22-26 July 2012

[8] T. K. Kristoffersen et. al," Optimal charging of electric drive vehicles in a market environment," in Applied Energy, vol. 88.5, pp. 1940-1948, 2011.

[9] T.Markel et. al, "Communication and control of electric drive vehicles supporting renewables," in Proc. 2009 IEEE Veh. Power Propulsion Conf., pp. 27-34.

[10] G.B. Shrestha, B.C.Chew, "Study on the optimization of chargedischarge cycle of EV batteries in the context of Singapore," in Proc. 2007 AUPEC Conf., pp. 1-7.

[11] M. Gonzalez Vaya, G. Andersson, "Optimal Bidding Strategy of a PEV Aggregator in Day-Ahead Electricity Markets Under Uncertainty," in Power Systems, IEEE Trans. on, vol. PP, no. 99, pp.1-11, 2014.

[12] L. Gan et. al, "Optimal decentralized protocol for EV charging," in Power Systems, IEEE Trans. on, vol. 28, no.2, pp. 940-451, 2013.

[13] J. Schmutzler et. al, "Cost Optimization of Energy Purchase for EV Fleets based on a Markovian EV Charging Model", in EVS27 Intl. BHFC EV Symposium, Barcelona, Spain, November 17 - 20, 2013.

[14] A. Soroudi and A. Keane, "Robust optimization based EV charging", in IEVC 2014 IEEE Intl., pp.1-6, December 17-19, 2014.

[15] R.F. Nelson, "Power requirements for batteries in hybrid EVs," in Power Sources, vol. 91, no. 1, pp. 2-26, Aug. 2000.

[16] A. Ben-Tal et. al, "Robust Optimization", Princeton Univ. Press, 2009.

[17] D. Bertsimas et. al, "Theory and applications of robust optimization", in SIAM review, pp. 464-501, vol.53, no 3., 2011.

[18] D. Bertsimas, J.N. Tsitsiklis, "Introduction to linear optimization," MIT, Athena Scientific, 1997.

[19] US National Grid, "Hourly electric supply charges, New York City" [Online]. Available: https://www.nationalgridus.com/niagaramohawk/ business/rates/5_hour_charge.asp [Sep 1, 2015]

[20] F. J. Massey, "The Kolmogorov-Smirnov test for goodness of fit," in Journ. of the Am. Stat. Assoc., pp.68-78, vol. 46, no.253, 1951.

[21] MathWorks, "Matlab R2014b documentation" [Online]. Available: http: //www.mathworks.com/help/stats/kstest.html [Sep 1, 2015]

[22] S. Boyd, L. Vandenberghe, "Localization and Cutting-Plane Methods" [Online]. Available: http://see.stanford.edu/materials/lsocoee364b/ 05-localization_methods_notes.pdf [Sep 1, 2015]
[23] M. Lubin, I. Dunning, "Computing in Operations Research Using Julia", in INFORMS J. on Computing, pp.238-248, vol. 27, no. 2, 2015.

[24] COIN-OR Projects, [Online]. Available: https://projects.coin-or.org/ Ipopt [Sep 1, 2015]

[25] COIN-OR Projects, [Online]. Available: https://projects.coin-or.org/ Bonmin [Sep 1, 2015]

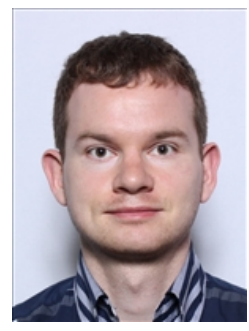

Nikita Korolko received his B.S. in 2010 and M.S degree in 2011 in Mathematics from Novosibirsk State University, Novosibirsk, Russia. He is a $\mathrm{PhD}$ student at the Operations Research Center at Massachusetts Institute of Technology, Cambridge, MA, USA. His current research interests include robust modeling and optimization, adaptive optimization and their applications to online problems. He held research intern positions at Mitsubishi Electric Research Laboratories, Cambridge, MA and Tesla Motors, Palo Alto, CA in 2014 and 2015, respectively.

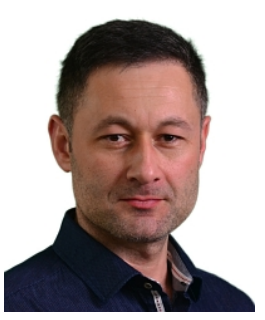

Zafer Sahinoglu received his MSc degree in 2001 and $\mathrm{PhD}$ in 2004 from New Jersey Institute of Technology in Biomedical Eng and Electrical Eng, respectively, and MBA in Operations Management from Massachusetts Institute of Technology Sloan School of Management in 2013. He worked at AT\&T's Shannon Research Labs, Florham Park, NJ as a visiting researcher in 2001, and then joined Mitsubishi Electric Research Labs. He has contributed and served in officer positions in numerous international standards in the areas of smartgrid, electric vehicles, indoor localization, wireless communications and sensor networks; and written two books published by Cambridge University Press. His current work includes remote sensing, robust modeling and optimization, battery modeling and energy big-data mining, and also development and integration of novel service businesses in productcentric companies. 